\title{
COMPOSIÇÃO CORPORAL E EXIGÊNCIAS NUTRICIONAIS DE CÁLCIO E FÓSFORO PARA GANHO EM PESO DE CORDEIROS ${ }^{1}$
}

\author{
EDINÉIA ALVES MOREIRA BAIÃO ${ }^{2}$ \\ JUAN RAMÓN OLALQUIAGA PEREZ ${ }^{3}$ \\ AFRANIO AFONSO FERRARI BAIÃ ${ }^{2}$ \\ LUCIANA CASTRO GERASEEV ${ }^{2}$ \\ ANDRÉ NUNES DE OLIVEIRA ${ }^{2}$ \\ JÚLIO CESAR TEIXEIRA ${ }^{3}$
}

\begin{abstract}
RESUMO - O experimento foi conduzido no Setor de Ovinocultura do Departamento de Zootecnia da UFLA, em Lavras, com o objetivo de determinar a composição corporal e estimar as exigências de cálcio e fósforo, para ganho em peso, de cordeiros puros Santa Inês e seus cruzamentos com Bergamácia, Ile de France e Texel. No experimento foram utilizados 48 cordeiros machos inteiros. Doze animais com peso médio de $15 \mathrm{~kg}$ foram abatidos no início do experimento para avaliar o conteúdo de cálcio e fósforo corporal, servindo como animais-referência para o método de abate comparativo. $\mathrm{O}$ restante dos animais foi abatido quando atingiram os pesos vivos de 25,35 e $45 \mathrm{~kg}$. A composição corporal de cálcio e fósforo foi estimada por meio de equações de regressão do logaritmo da quantidade dos minerais presentes no corpo vazio em função do logaritmo do pe-
\end{abstract}

so do corpo vazio. As exigências líquidas de cálcio e fósforo para o ganho em peso foram estimadas a partir da derivação das equações de predição da composição corporal. A estimativa da composição corporal por quilo de peso corporal vazio para animais de 15 a $45 \mathrm{~kg}$ de peso vivo foi: 14,641 a 11,637 $\mathrm{g}$ de $\mathrm{Ca}$ para os animais Santa Inês e 12,537 a 10,778 g de Ca para os demais grupos genéticos. No caso do fósforo, não houve diferenças entre os grupos genéticos, e os valores variaram de 7,892 a 6,767 g de P. As exigências líquidas por quilograma de ganho de peso vivo para animais de 15 a 45 $\mathrm{kg}$ de PV foram, respectivamente: 9,50 $\mathrm{g}$ a 7,56 $\mathrm{g}$ de $\mathrm{Ca}$ para animais puros Santa Inês; 8,78 g a 7,54 g para os demais animais. No caso do fósforo, as exigências para animais de $15 \mathrm{~kg}$ foi de $5,5 \mathrm{~g}$ de $\mathrm{P}$ e para animais com $45 \mathrm{~kg}$ foi de $4,7 \mathrm{~g}$ de $\mathrm{P}$.

TERMOS PARA INDEXAÇÃO: Ovinos, crescimento, requerimentos, minerais.

\section{BODY COMPOSITION AND NUTRICIONAL REQUERIMENTS OF CALCIUM AND PHOSPHORUS FOR WEIGHT GAIN OF LAMBS}

\begin{abstract}
The experiment was conducted in the Sheep Division of Animal Science Department at UFLA in Lavras with the objective to determine the body composition and to estimate the calcium and phosphorus requirements for weight gain of Santa Inês pure lambs and their crossings with Bergamácia, Ile de France and Texel breeds. It was used 48 non castrated male lambs. Twelve lambs with average weight of 15 $\mathrm{kg}$ were slaughtered in the beginning of the experiment to evaluate the calcium and phosphorus body content by using as reference animals for the comparative
\end{abstract}

slaughtered method. The animals remaining were slaughtered when they reached 25,35 and $45 \mathrm{~kg}$ of live weight, respectively. The calcium and phosphorus body composition was estimated by prediction equations, obtained by the regression of the mineral empty body amount logarithm in function of empty body weight logarithm. The net requirements of calcium and phosphorus for gaining in weight were estimated from the derivation of the prediction equations of the body composition. The estimate of the body composition per kilogram of empty body weight for animals from 15 to

1. Extraído da dissertação de mestrado apresentada pelo primeiro autor à UNIVERSIDADE FEDERAL DE LAVRAS/UFLA - Caixa Postal 37 - 37200-000 - Lavras, MG.

2. Alunos do Curso de Pós-Graduação em Zootecnia/UFLA. eambaiao@yahoo.com.br, Fone: (35) 3821-1680

3. Professores da UFLA. 
$45 \mathrm{~kg}$ of live weight was: 14.641 to $11.637 \mathrm{~g}$ of $\mathrm{Ca}$ for the Santa Inês animals and 12.537 to $10.778 \mathrm{~g}$ of $\mathrm{Ca}$ for the other genetic groups. In the case of the phosphorus there were not differences among the genetic groups, and the values varied from 7.892 to $6.767 \mathrm{~g}$ of $\mathrm{P}$. The net requirements per kilogram of live weight gain for

INDEX TERMS: Sheep, growth, requeriments, minerals.

\section{INTRODUÇÃO}

O cálcio e o fósforo devem estar disponíveis na dieta em quantidades e proporções adequadas para atender às necessidades dos animais em relação à idade, raça, categoria ou situação fisiológica e sistema de produção adotado.

Vários fatores influenciam o requerimento de minerais, incluindo natureza e nível de produção, idade, nível e forma química do elemento nos ingredientes da dieta, inter-relações com outros nutrientes, raça e adaptação do animal ao meio em que vive (MCDOWELL, 1999). O AFRC (1991) ressalta que além do suprimento adequado de minerais, são necessários níveis adequados de proteína e energia para que ocorra desenvolvimento normal dos ossos.

A importância de se estudar a composição química do corpo e do ganho em peso está no fato de os mesmos constituírem parâmetros indispensáveis nas avaliações de programas de nutrição e nas determinações das exigências nutricionais.

A exigência de macrominerais para ganho em peso tem sido estimada pelo método fatorial. Esse método baseia-se nas quantidades líquidas depositadas no corpo do animal para atender ao ganho em peso (crescimento e a engorda), gestação, lactação e ao crescimento de lã em ovinos. A essas exigências líquidas são acrescidas as quantidades necessárias para atender às perdas inevitáveis do corpo e ao metabolismo basal do animal, ou seja, exigências líquidas de manutenção. A soma das frações de mantença e produção vão constituir a exigência líquida total, a qual, corrigida por um coeficiente de absorção do elemento inorgânico no aparelho digestivo do animal, resulta na exigência dietética do animal (ARC, 1980).

O AFRC (1991), de posse de novos dados e informações disponíveis, adotou equações baseadas no crescimento ósseo para estimar as exigências de cálcio e fósforo, e considerou que a concentração desses elementos no corpo diminui à medida que o animal tornase adulto. Para animais com $20 \mathrm{~kg}$ e $40 \mathrm{~kg}$ de PV, os valores recomendados variam de 10,6 a 8,7 $\mathrm{g}$ de $\mathrm{Ca}$ por $\mathrm{kg}$ de PV e 6,2 a 5,3 g de P por kg de PV. animals from 15 to $45 \mathrm{~kg}$ of $\mathrm{LW}$ were, respectively: $9.50 \mathrm{~g}$ to $7.56 \mathrm{~g}$ of $\mathrm{Ca}$ for Santa Inês pure animals; 8.78 $\mathrm{g}$ to $7.54 \mathrm{~g}$ for the other animals. In the case of the phosphorus, this requeriments for animals of $15 \mathrm{~kg}$ of LW was of $5.5 \mathrm{~g}$ of P and for animals with $45 \mathrm{~kg}$ of LW was of $4.7 \mathrm{~g}$ of P.

No Brasil, os cálculos e o balanceamento de ração para ovinos têm sido baseados nas tabelas do National Research Council (NRC) e do Agricultural Research Council (ARC), tornando-se limitantes, uma vez que essas recomendações expressam exigências de ovinos lanados em países de clima temperado, deixando dúvidas quanto ao uso desses requerimentos para ovinos criados em regiões tropicais (SILVA, 1995). Desse modo, torna-se necessário estabelecer as necessidades alimentares de ovinos lanados e deslanados criados nas condições brasileiras, para obtenção de um sistema nutricional mais eficiente e econômico que atenda aos reais requerimentos.

Objetivou-se com este trabalho determinar a composição corporal e estimar as exigências de cálcio e fósforo para ganho em peso em cordeiros Santa Inês e seus cruzamentos com Bregamácia, Ile de France e Texel criados na região sul de Minas Gerais e sua comparação com as tabelas de exigências rotineiramente usadas no nosso país.

\section{MATERIAL E MÉTODOS}

O trabalho foi realizado no Setor de Ovinocultura do Departamento de Zootecnia da Universidade Federal de Lavras, na cidade de Lavras - Minas Gerais.

Foram utilizados 48 cordeiros machos inteiros provenientes dos cruzamentos entre ovinos puros Santa Inês x Santa Inês (SI),e F1- Bergamácia x Santa Inês (BE); F1- Ilê de France x Santa Inês (IF) e, F1 Texel x Santa Inês (TE); com peso vivo médio inicial de $15 \mathrm{~kg}$.

Esses animais foram divididos em quatro grupos: doze animais escolhidos aleatoriamente, dos quais três de cada grupo genético foram abatidos no início do experimento para avaliação do conteúdo de cálcio e fósforo corporal, servindo como animais-referência para o método de abate comparativo.

A dieta experimental (Tabela 1) foi balanceada de forma a atender às exigências nutricionais de proteína, energia metabolizável e minerais, segundo as recomendações do ARC (1980), sendo fornecida duas vezes ao dia, às oito e às 16 horas. $\mathrm{O}$ consumo foi medido 
diariamente, pela pesagem das quantidades fornecidas e rejeitadas. Os animais foram pesados semanalmente sempre na parte da manhã antes de receber a primeira alimentação.

Ao atingirem o peso médio inicial de $15 \mathrm{~kg}$, doze animais foram abatidos para servirem de animaisreferência. Os demais foram confinados em gaiolas individuais de $1,3 \times 1,0 \mathrm{~m}$ com cocho para sal mineralizado, comedouro e bebedouro, e foram abatidos quando alcançaram os pesos de: 25,35 e $45 \mathrm{~kg}$, respectivamente. $\mathrm{O}$ abate foi feito por sangramento mediante corte da carótida e da jugular dos animais, sendo o sangue coletado, pesado e congelado para análises posteriores. Após a coleta do sangue, retiraram-se o trato digestivo, bexiga e vesícula biliar, sendo seus conteúdos eliminados para determinação do peso corporal vazio. O corpo do animal foi subdividido em partes menores, juntamente com o aparelho digestivo, vísceras, sangue, cabeça, patas e pele, sendo acondicionados em sacos plásticos e, em seguida, congelados. Esse material congelado foi serrado e moído em cutter de 30 H.P. e 1775 rpm; em seguida, foi homogeneizado e novamente moído, sendo acondicionado em sacos plásticos para serem congelados. Esse procedimento foi repetido por três vezes para serem retiradas as amostras para as análises químicas. As análises químicas foram efetuadas segundo a metodologia descrita por Silva (1998). As amostras referentes ao corpo dos animais foram pré-secas em estufa a $65^{\circ} \mathrm{C}$ com circulação de ar por 72 horas. Após a secagem, esse material foi desengordurado em aparelho Soxhlet e triturado.

As análises para determinação dos macrominerais nas amostras dos ingredientes da ração, na ração e na matéria seca desengordurada do corpo do animal, foram efetuadas por digestão ácida com ácido nítrico e ácido perclórico, obtendo-se, dessa forma, a solução mineral. Em seguida, foram feitas as diluições para determinação do cálcio e fósforo. $\mathrm{O}$ cálcio foi determinado adicionando-se cloreto de estrôncio e as leituras, tomadas em espectrofotômetro de absorção atômica. Já o fósforo foi determinado por redução do complexo fósforo-molibdato e as leituras foram tomadas em espectofotômetro (colorímetro).

TABELA 1 - Composição da dieta experimental expressa em porcentagem da matéria seca.

\begin{tabular}{|c|c|c|c|c|c|}
\hline Ingredientes & $\mathbf{M S}^{\mathbf{1}}$ & $\begin{array}{c}\mathbf{E M}^{2} \\
(\text { Kcal } / \mathbf{k g})\end{array}$ & $\mathbf{P B}^{1}$ & $(\%)$ & $(\%)$ \\
\hline Milho moído & 66,23 & 2087 & 6,49 & 0,022 & 0,196 \\
\hline Farelo de soja & 12,37 & 394 & 6,28 & 0,053 & 0,097 \\
\hline Feno de coast cross & 20,25 & 395 & 2,44 & 0,120 & 0,080 \\
\hline Calcário calcítico & 0,85 & - & - & 0,360 & - \\
\hline Sal comum & 0,25 & - & - & - & - \\
\hline Supl. Microminerais ${ }^{3}$ & 0,01 & - & - & - & - \\
\hline Supl. Vitamínico ${ }^{3}$ & 0,04 & - & - & - & - \\
\hline TOTAL & 100,00 & 2876 & 15,21 & 0,501 & 0,373 \\
\hline
\end{tabular}

${ }^{1}$ Análises realizadas no Laboratório de Nutrição Animal do Departamento de Zootecnia ${ }^{2}$ NRC (1985)

${ }^{3}$ Suplemento Microminerais e Vitamínico (nutriente/Kg de suplemento): vit. A 2.500.000 UI, Vit.D3 500.000UI, Vit. E 3000mg, Tiamina $750 \mathrm{mg}$, Riboflavina 1000mg, VitB12 $2800 \mathrm{mcg}$, Niacina $500 \mathrm{mg}$, Selênio $150 \mathrm{mg}$, Iodo 1000mg, Cobalto 600mg, Ferro $35000 \mathrm{mg}$, Cobre $20000 \mathrm{mg}$, Manganês 49000mg, Zinco 75000 mg. 
O delineamento experimental foi inteiramente casualizado (DIC), sendo feita a análise de regressão das quantidades dos macrominerais presentes no corpo vazio em função do peso corporal vazio. Os resultados foram interpretados estatisticamente por meio de análise de variância e regressão, utilizando-se o Sistema de Análises Estatísticas e Genéticas - SAEG (EUCLIDES e SILVA, 1979). O modelo utilizado foi: $Y_{i j}=\mu+b i x i j+$ eij $\mathrm{Y}_{\mathrm{ij}}=$ logaritmo da quantidade de macromineral presente no corpo vazio;

$\mu=$ efeito da média;

$\mathrm{b}_{\mathrm{i}}=$ coeficiente de regressão;

$\mathrm{X}_{\mathrm{ij}}=$ logaritmo do peso do corpo vazio;

$\mathrm{e}_{\mathrm{ij}}=$ erro aleatório

As equações de predição da composição corporal foram determinadas segundo a metodologia proposta pelo ARC (1980), pela regressão do logaritmo da quantidade dos minerais presentes no corpo vazio dos animais, em função do peso corporal vazio:

$$
\log y=a+b \log x
$$

em que:

Log y = Logaritmo do conteúdo total do macromineral no corpo vazio

$\mathrm{a}=$ intercepto

$\log x=$ Logaritmo do peso corporal vazio

$\mathrm{b}=$ Coeficiente de regressão do conteúdo do macromineral em função do peso corporal vazio.

As exigências líquidas para o ganho em peso corporal vazio foram estimadas pela derivação das equações de regressão de predição da composição corporal, obtendo-se equações do tipo y' $=$ b. $10^{\mathrm{a}} . \mathrm{PCV}^{(\mathrm{b}-1)}$. As exigências líquidas desses minerais para o ganho de peso vivo foram calculadas dividindo-se as exigências líquidas de ganho corporal vazio pelo fator 1,25 , calculado a partir das equações de conversão do peso corporal vazio em peso vivo. Para as exigências líquidas de mantença, foram utilizados os valores propostos pelo ARC (1980).

Para o cálculo das exigências dietéticas totais, foram utilizados os valores de perdas endógenas de $16,0 \mathrm{mg} / \mathrm{kg}$ PV para o cálcio e $14,0 \mathrm{mg} / \mathrm{kg} \mathrm{PV}$ de fósforo, sendo a disponibilidade na dieta de 68 e $73 \%$, respectivamente, segundo estimativas do (ARC, 1980).

Nas equações de regressão do logaritmo do conteúdo corporal de cálcio e fósforo, em função do logaritmo do peso corporal vazio (PCV), para os quatro grupos genéticos estudados (SI, BE, IF e TE), com pesos variando dos 15 aos $45 \mathrm{~kg}$ de peso vivo, foi aplicada a análise de comparação de equações lineares
(SNEDECOR e COCHRAN, 1967), para detectar possíveis diferenças entre os grupos genéticos.

\section{RESULTADOS E DISCUSSÃO}

Na tabela 2 estão apresentados os resultados médios do peso vivo de abate, peso corporal vazio (PCV) e a composição corporal em cálcio e fósforo encontrados nos cordeiros puros SI e, BE, IF e TE nos pesos 15, 25, 35 e $45 \mathrm{~kg}$ de peso vivo.

Analisando a Tabela 2, observa-se que houve um decréscimo na quantidade dos minerais no corpo vazio, em função do aumento do peso vivo dos mesmos. Esse decréscimo no conteúdo de $\mathrm{Ca}$ e $\mathrm{P}$ também foi observado com ovinos (GERASEEV et al. 2000; TRINDADE, 2000); bovinos (SIGNORETTI, 1998; PAULINO et al., 1999) e caprinos (RESENDE, 1989; RIBEIRO, 1995).

As equações do AFRC (1991) também estimaram decréscimos nas concentrações de Ca e $\mathrm{P}$ no corpo vazio, em função do aumento do peso vivo dos animais. A possível explicação para tal fato provavelmente deve estar relacionada ao aumento do teor de gordura e à redução no crescimento ósseo desses animais, com a elevação do peso corporal vazio. Todavia, o ARC (1980) considera a deposição desses minerais constante e estima as seguintes concentrações corporais: $11 \mathrm{~g}$ de $\mathrm{Ca}$ e $6 \mathrm{~g}$ de $\mathrm{P} / \mathrm{kg}$ de peso corporal vazio.

Pela análise de comparação de equações de predição da composição corporal, verifica-se que há diferenças significativas para os grupos F1 e para os animais puros Santa Inês nas equações de predição da composição corporal em cálcio. Assim, adotaram-se uma equação geral para os animais F1 e uma equação para os animais puros Santa Inês. Quanto ao fósforo, a análise de comparação de equações mostrou não haver diferenças significativas nas equações estimadas para os diferentes grupos genéticos; portanto, adotou-se uma equação geral obtida com todos os animais para estimar a quantidade de P no corpo vazio dos mesmos (Tabela 3 ).

Os coeficientes de determinação, todos significativos $(p<0,05)$, mostram o ajuste elevado do modelo para estimar a composição dos animais em estudo.

Com base nas equações da Tabela 3, foi estimado o conteúdo de cálcio e fósforo no corpo vazio em função do peso corporal vazio nos cordeiros (Tabela 4).

Para a predição da composição do ganho em cálcio e fósforo, derivaram-se as equações de predição da composição corporal, obtendo-se, então, equações que permitiram estimar as exigências líquidas desses minerais para o ganho em peso corporal vazio (Tabela 5). 
TABELA 2 - Valores médios da composição corporal de cordeiros puros Santa Inês x Santa Inês e, os F1- Bergamácia x Santa Inês, Ile de France x Santa Inês e Texel x Santa Inês abatidos em diferentes pesos vivos.

\begin{tabular}{llcccc}
\hline \multirow{2}{*}{ Grupo genético } & \multirow{2}{*}{ Item } & \multicolumn{3}{c}{ Peso de abate (kg) } \\
\cline { 3 - 6 } & & $\mathbf{1 5}$ & $\mathbf{2 5}$ & $\mathbf{3 5}$ & $\mathbf{4 5}$ \\
\cline { 3 - 6 } Santa Inês x (SI) & M.S.(kg) & $37,94 \pm 2,663$ & $41,57 \pm 0,804$ & $42,88 \pm 0,825$ & $44,56 \pm 0,937$ \\
& Gordura(\%MN) & $10,15 \pm 0,473$ & $14,16 \pm 0,107$ & $19,09 \pm 0,268$ & $22,16 \pm 2,057$ \\
& Cálcio(\%MN) & $1,378 \pm 0,117$ & $1,175 \pm 0,019$ & $1,156 \pm 0,015$ & $1,103 \pm 0,011$ \\
& Fósforo(\%MN) & $0,806 \pm 0,082$ & $0,718 \pm 0,066$ & $0,710 \pm 0,006$ & $0,598 \pm 0,005$ \\
\hline \multirow{5}{*}{ Bergamácia x (SI) } & M.S.(kg) & $38,41 \pm 1,226$ & $41,33 \pm 1,079$ & $42,71 \pm 0,705$ & $44,37 \pm 1,406$ \\
& Gordura(\%MN) & $10,31 \pm 0,430$ & $14,01 \pm 0,282$ & $18,95 \pm 0,514$ & $22,07 \pm 0,836$ \\
& Cálcio(\%MN) & $1,266 \pm 0,096$ & $1,149 \pm 0,053$ & $1,133 \pm 0,031$ & $1,074 \pm 0,038$ \\
& Fósforo(\%MN) & $0,790 \pm 0,040$ & $0,710 \pm 0,025$ & $0,702 \pm 0,020$ & $0,673 \pm 0,025$ \\
\hline \multirow{3}{*}{ Ilê deFrance x (SI) } & M.S.(kg) & $38,91 \pm 0,944$ & $41,97 \pm 0,229$ & $43,03 \pm 1,664$ & $47,24 \pm 0,783$ \\
& Gordura(\%MN) & $11,48 \pm 0,445$ & $14,41 \pm 0,197$ & $19,12 \pm 0,034$ & $24,26 \pm 0,343$ \\
& Cálcio(\%MN) & $1,192 \pm 0,072$ & $1,150 \pm 0,019$ & $1,113 \pm 0,018$ & $1,070 \pm 0,013$ \\
& Fósforo(\%MN) & $0,713 \pm 0,046$ & $0,713 \pm 0,023$ & $0,699 \pm 0,008$ & $0,673 \pm 0,024$ \\
\hline & M.S.(kg) & $38,73 \pm 1,626$ & $41,81 \pm 0,394$ & $42,67 \pm 0,446$ & $45,58 \pm 0,357$ \\
& Gordura(\%MN) & $11,45 \pm 0,240$ & $14,18 \pm 0,858$ & $19,07 \pm 0,297$ & $23,99 \pm 1,018$ \\
& Cálcio(\%MN) & $1,216 \pm 0,136$ & $1,141 \pm 0,61$ & $1,123 \pm 0,036$ & $1,071 \pm 0,051$ \\
& Fósforo(\%MN) & $0,756 \pm 0,066$ & $0,713 \pm 0,023$ & $0,699 \pm 0,008$ & $0,672 \pm 0,024$ \\
\hline
\end{tabular}

TABELA 3 - Equações de regressão para estimar o peso de corpo vazio (g) em função do peso vivo(g) e composição corporal de cálcio e fósforo presentes no corpo vazio em função do peso corporal vazio para cordeiros dos 15 aos $45 \mathrm{~kg}$ de PV em quatro grupos genéticos (SI, BE, IF e TE).

\begin{tabular}{lcc}
\hline \multicolumn{1}{c}{ Item } & \multicolumn{1}{c}{ Equação } & $\mathbf{R}^{\mathbf{2}}$ (\%) \\
\hline Peso corp. Vazio(g) & $\mathrm{PCV}=-1,852059+0,848100 \mathrm{PV}$ & $\mathrm{R}^{2}=96,79$ \\
Cálcio (g) SI & $\log \mathrm{Ca}=-1,06607+0,809636 \mathrm{Log} \mathrm{PCV}$ & $\mathrm{R}^{\mathbf{2}}=98,27$ \\
Cálcio (g) BE, IF, TE ${ }^{2}$ & $\log \mathrm{Ca}=-1,39572+0,874616 \mathrm{Log} \mathrm{PCV}$ & $\mathrm{R}^{2}=98,59$ \\
Fósforo (g) SI,BE,IF,TE & $\log \mathrm{P}=-1,58862+0,872599 \log \mathrm{PCV}$ & $\mathrm{R}^{2}=98,28$ \\
\hline
\end{tabular}

1Equação de regressão para os animais puros Santa Inês (SI) para o conteúdo corporal de Ca; ${ }^{2}$ Equação de regressão para os animais F1- (Bergamácia (BE), Ilê de France (IF), Texel (TE) para o conteúdo corporal de Ca;

${ }^{3}$ Equação de regressão para os grupos genéticos Santa Inês (SI),e F1- Bergamácia (BE), Ilê de France (IF), Texel (TE) para o conteúdo corporal de $P$. 
TABELA 4 - Estimativa do conteúdo de cálcio e fósforo no corpo vazio em função do peso corporal vazio em cordeiros SI, e BE, IF e TE.

\begin{tabular}{cccccc}
\hline \multirow{2}{*}{$\mathbf{P V}$} & \multirow{k}{*}{$\begin{array}{c}\text { PCV } \\
(\mathbf{k g})\end{array}$} & $\mathbf{C a}(\mathbf{S I})$ & $\mathbf{C a}(\mathbf{B E}, \mathbf{I F}, \mathbf{T E})$ & $\mathbf{P}(\mathbf{S I} \mathbf{e}$ BE, IF, TE) \\
\cline { 4 - 6 } & 10,87 & 14,641 & 12,537 & 7,892 \\
\hline 15 & 15,10 & 13,753 & 12,031 & 7,568 \\
20 & 19,35 & 13,118 & 11,663 & 7,332 \\
25 & 23,60 & 12,632 & 11,376 & 7,150 \\
30 & 27,83 & 12,242 & 11,143 & 7,000 \\
35 & 33,92 & 11,789 & 10,870 & 6,826 \\
40 & 36,31 & 11,637 & 10,778 & 6,767 \\
45 & & 12,83 & 11,48 & 7,21 \\
\hline Média & & 11,00 & 11,00 & 6,00 \\
\hline ARC (1980) & & &
\end{tabular}

TABELA 5 - Equações derivadas para estimar exigências líquidas de cálcio e fósforo.

\section{Cálcio para os animais (SI) ${ }^{1}$}

Cálcio para os animais (BE), (IF), (TE)

Fósforo para os animais (SI), (BE), (IF), (TE)

$$
\begin{gathered}
Y^{\prime}=0,06955 \cdot \mathrm{PCV}^{-0,190164} \\
\mathrm{Y}^{\prime}=0,035164 \cdot \mathrm{PCV}^{-0,125384} \\
\mathrm{Y}^{\prime}=0,0225 \cdot \mathrm{PCV}^{-0,127401}
\end{gathered}
$$

\section{${ }^{1}$ SI- Santa Inês puros; BE- Bergamácia x Santa Inês; IF- Ile de France x Santa Inês; TE- Texel x Santa Inês.}

$\mathrm{Na}$ Tabela 4, analisando a composição corporal de cálcio entre os grupos genéticos, verificou-se que os animais puros Santa Inês apresentaram valores superiores quando comparados com os F1- Bergamácia, Ile de France e Texel. Essa diferença na concentração de cálcio corporal para animais puros Santa Inês e os F1 provavelmente é devida principalmente às diferenças existentes na proporção de ossos na carcaça, uma vez que $98 \%$ do conteúdo de cálcio do corpo estão nos ossos e também na variação da concentração de gordura corporal, que ocorreu em função da idade, raça, grupo genético, sexo, manejo alimentar e condições climáticas.

Os valores médios estimados nesta pesquisa para animais puros SI foi de $12,83 \mathrm{~g}$ de $\mathrm{Ca} / \mathrm{kg}$ e de $11,48 \mathrm{~g}$ por kg para os BE, IF e TE quando comparam-se os valores médios de $\mathrm{Ca}$, estimados por este trabalho para os animais F1, observa-se que estes valores estão próximos dos valores recomendados pelo ARC (1980), que é de $11 \mathrm{~g} / \mathrm{kg}$ de PCV.
Os animais Santa Inês foram mais exigentes em cálcio que os BE, IF e TE. Quando se comparam as exigências líquidas de ganho em PCV (Peso Corporal Vazio) de cálcio, obtidas nesta pesquisa, com os valores recomendados pelo ARC (1980), observa-se que essas são aproximadamente $8 \%$ maiores para os animais SI, com $15 \mathrm{~kg}$ de peso vivo e $16,52 \%$ menores para animais com $45 \mathrm{~kg}$ de peso vivo. Entretanto, para os animais BE, IF, TE com 15 $\mathrm{kg}$ de PV, os valores estimados para o cálcio foram semelhantes aos valores recomendados pelo ARC (1980).

O AFRC (1991) considera um requerimento de fósforo para cordeiros com $40 \mathrm{Kg}$ de $5,3 \mathrm{~g} / \mathrm{kg}$ de PV. Esse valor é aproximadamente 11,32\% inferior aos valores estimados neste trabalho para animais na mesma faixa de peso. Isso provavelmente ocorreu devido às diferenças na composição corporal dos animais estudados e às condições climáticas. 
Quanto aos valores encontrados nesta pesquisa, para a composição do ganho em peso corporal vazio para o fósforo com todos os animais do experimento com peso vivo de $45 \mathrm{~kg}$, observa-se que esses valores são semelhantes aos valores recomendados pelo ARC (1980).

É importante ressaltar que a retenção de $\mathrm{Ca}$ e $\mathrm{P}$ depende da composição do ganho. Maiores deposições de gordura reduzem as deposições desses minerais e, conseqüentemente, seus requerimentos pelos animais, já que as concentrações de cálcio e fósforo no tecido adiposo são insignificantes, com maiores concentrações nos músculos e ossos. Portanto, fatores como sexo, grupo genético, peso e idade dos animais influenciam os requerimentos desses minerais.

Nas Tabelas 7 a 9 são apresentadas as estimativas das exigências líquidas e dietéticas de cálcio e fósforo dos cordeiros puros Santa Inês e os F1- Bergamácia, Ilê de France e Texel, calculadas conforme a metodologia citada no item Material e Métodos.

TABELA 6 - Estimativa da concentração de cálcio e fósforo corporal do ganho em peso de corpo vazio de cordeiros SI e, BE, IF e TE em função do peso corporal vazio.

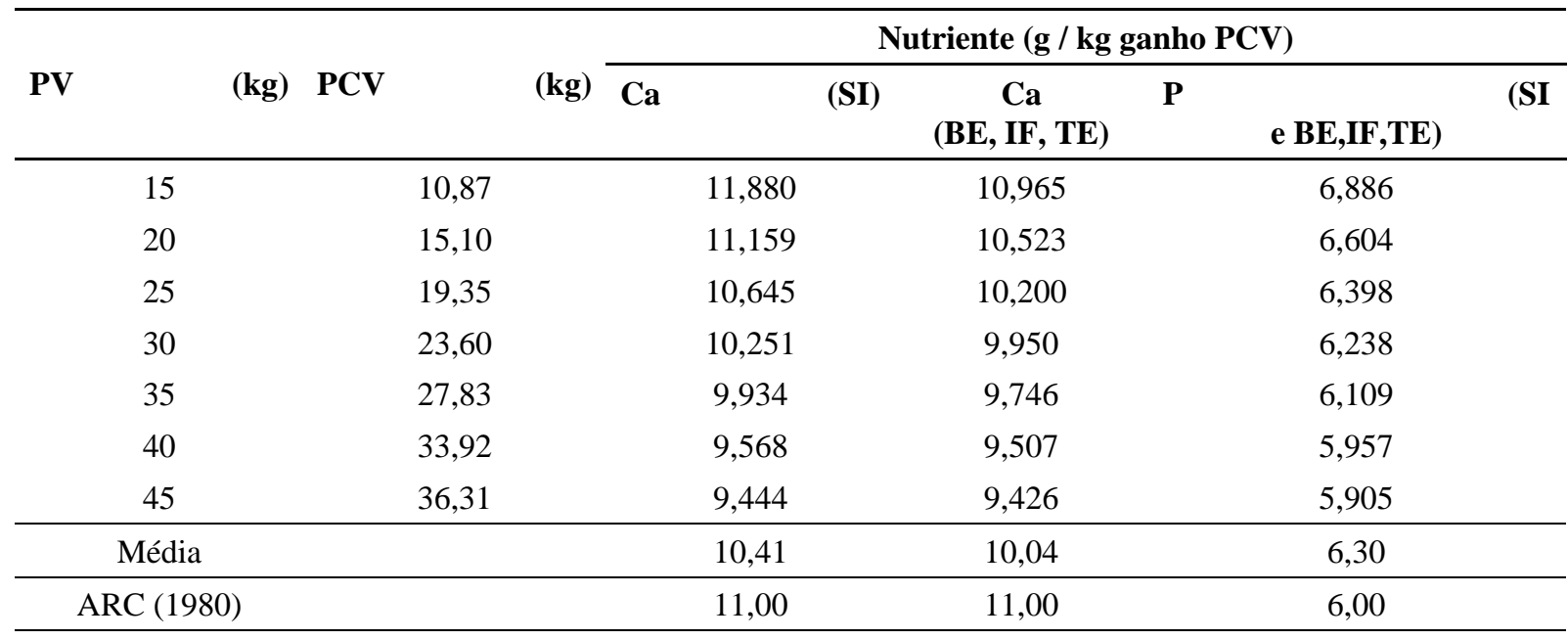

TABELA 7 - Estimativas das exigências líquidas e dietéticas de cálcio para mantença e ganho em peso vivo (g/animal/dia) em animais puros Santa Inês.

\begin{tabular}{ccccccccc}
\hline \multirow{2}{*}{$\begin{array}{c}\text { Peso vivo } \\
\text { (kg) }\end{array}$} & \multicolumn{7}{c}{ Ganho Diário(g) } \\
\cline { 2 - 8 } & \multicolumn{7}{c}{ Exigência Líquida } \\
\cline { 2 - 8 } & Mantença & $\mathbf{1 0 0}$ & $\mathbf{2 0 0}$ & $\mathbf{3 0 0}$ & Mantença & $\mathbf{1 0 0}$ & $\mathbf{2 0 0}$ & $\mathbf{3 0 0}$ \\
\hline & 0,240 & 0,950 & 1,900 & 2,850 & 0,353 & 1,397 & 2,794 & 4,191 \\
\hline 15 & 0,320 & 0,893 & 1,786 & 2,619 & 0,470 & 1,313 & 2,626 & 3,940 \\
20 & 0,400 & 0,851 & 1,702 & 2,553 & 0,588 & 1,251 & 2,502 & 3,753 \\
25 & 0,480 & 0,820 & 1,640 & 2,460 & 0,706 & 1,205 & 2,410 & 3,615 \\
30 & 0,560 & 0,795 & 1,590 & 2,385 & 0,823 & 1,169 & 2,338 & 3,507 \\
35 & 0,640 & 0,765 & 1,530 & 2,295 & 0,941 & 1,125 & 2,250 & 3,375 \\
40 & 0,720 & 0,756 & 1,512 & 2,268 & 1,059 & 1,111 & 2,222 & 3,333 \\
45 & & & & & & & & \\
\end{tabular}

Ciênc. agrotec., Lavras. V.27, n.6, p.1370-1379, nov./dez., 2003 
TABELA 8 - Estimativas das exigências líquidas e dietéticas de cálcio para mantença e ganho em peso vivo (g/animal/dia) em animais BE, IF e TE.

\begin{tabular}{ccccccccc}
\hline \multirow{2}{*}{$\begin{array}{c}\text { Peso vivo } \\
\text { (kg) }\end{array}$} & \multicolumn{7}{c}{ Ganho Diário(g) } \\
\cline { 2 - 9 } & \multicolumn{7}{c}{ Exigência Líquida } \\
\cline { 2 - 10 } & Mantença & $\mathbf{1 0 0}$ & $\mathbf{2 0 0}$ & $\mathbf{3 0 0}$ & Mantença & $\mathbf{1 0 0}$ & $\mathbf{2 0 0}$ & $\mathbf{3 0 0}$ \\
\hline 15 & 0,240 & 0,878 & 1,756 & 2,634 & 0,353 & 1,291 & 2,582 & 3,873 \\
20 & 0,320 & 0,842 & 1,684 & 2,526 & 0,470 & 1,238 & 2,476 & 3,714 \\
25 & 0,400 & 0,816 & 1,632 & 2,448 & 0,588 & 1,200 & 2,400 & 3,600 \\
30 & 0,480 & 0,796 & 1,592 & 2,388 & 0,706 & 1,170 & 2,340 & 3,510 \\
35 & 0,560 & 0,780 & 1,560 & 2,340 & 0,823 & 1,147 & 2,294 & 3,441 \\
40 & 0,640 & 0,760 & 1,520 & 2,280 & 0,941 & 1,118 & 2,236 & 3,354 \\
45 & 0,720 & 0,754 & 1,508 & 2,262 & 1,059 & 1,109 & 2,218 & 3,327 \\
\hline
\end{tabular}

TABELA 9 - Estimativas das exigências líquida e dietéticas de fósforo para a mantença e ganho em peso vivo (g/animal/dia) em animais SI e BE, IF e TE.

\begin{tabular}{ccccccccc}
\hline \multirow{2}{*}{$\begin{array}{c}\text { Peso vivo } \\
\text { (kg) }\end{array}$} & \multicolumn{7}{c}{ Ganho Diário(g) } \\
\cline { 2 - 9 } & \multicolumn{7}{c}{ Exigência Líquida } \\
\cline { 2 - 9 } & Mantença $(\mathbf{g})$ & $\mathbf{1 0 0}$ & $\mathbf{2 0 0}$ & $\mathbf{3 0 0}$ & Manimal / dia) \\
\hline 15 & 0,210 & 0,550 & 1,100 & 1,650 & 0,288 & 0,753 & 1,506 & 2,259 \\
20 & 0,280 & 0,528 & 1,056 & 1,584 & 0,383 & 0,723 & 1,446 & 2,169 \\
25 & 0,350 & 0,512 & 1,024 & 1,536 & 0,479 & 0,701 & 1,402 & 2,103 \\
30 & 0,420 & 0,499 & 0,998 & 1,497 & 0,575 & 0,684 & 1,368 & 2,052 \\
35 & 0,490 & 0,488 & 0,976 & 1,464 & 0,671 & 0,668 & 1,336 & 2,004 \\
40 & 0,560 & 0,477 & 0,954 & 1,431 & 0,767 & 0,653 & 1,306 & 1,959 \\
45 & 0,630 & 0,472 & 0,944 & 1,416 & 0,863 & 0,647 & 1,294 & 1,941 \\
\hline
\end{tabular}

Analisando as exigências dietéticas de ganho de cálcio para o grupo SI, obtidas nesta pesquisa, com os valores recomendados pelo ARC (1980), observa-se que essas são aproximadamente $15,62 \%$ menores para animais com $15 \mathrm{~kg}$ de peso vivo e $38,88 \%$ menores para os animais com $45 \mathrm{~kg}$ de peso vivo. Já para os animais BE, IF e TE, os valores obtidos para o cálcio são aproximadamente $21,87 \%$ menores que os valores recomendados pelo ARC (1980), para animais com 15 $\mathrm{kg}$ de peso vivo.

Ao contrário do ARC (1980), que considera a concentração de cálcio e fósforo no ganho de peso constante durante o crescimento e engorda do animal, este trabalho encontrou queda nas quantidades de cálcio e fósforo por unidade de ganho de peso, devido à 
redução da taxa de crescimento ósseo e ao aumento da deposição de gordura corporal.

Com relação às recomendações preconizadas pelo AFRC (1991), que recomenda aproximadamente 4,0 g de Ca para animais com $15 \mathrm{~kg}$ e 3,75 g de Ca para animais com $45 \mathrm{~kg}$ de peso vivo, as exigências dietéticas de cálcio para animais SI encontradas neste trabalho foram $3,85 \%$ maiores para animais com $15 \mathrm{~kg}$ de peso vivo e $12,5 \%$ menores para animais com $45 \mathrm{~kg}$ de peso vivo, apresentando uma taxa de ganho de $200 \mathrm{~g} / \mathrm{dia}$.

Comparando as exigências dietéticas de fósforo estimadas neste trabalho para cordeiros SI e BE, IF e TE, com as exigências propostas pelo ARC (1980), observa-se que essas são aproximadamente $11,76 \%$ inferiores às citadas por esse comitê para cordeiros com $15 \mathrm{~kg}$ de peso vivo e $38,1 \%$ inferiores para animais com $45 \mathrm{~kg}$ de PV para um ganho diário de $200 \mathrm{~g} / \mathrm{dia}$.

As estimativas da composição corporal e exigências líquidas de cálcio e fósforo para cordeiros SI e BE, IF e TE, estimadas neste trabalho, diferiram dos valores propostos pelo ARC (1980). Todavia, essas mesmas estimativas guardam relação estreita com as estimativas preconizadas pelo AFRC (1991). Essas diferenças existentes entre os valores da composição corporal e as exigências em cálcio e fósforo, para animais Santa Inês e, os F1- Bergamácia, Ile de France e Texel, estimadas neste estudo em relação aos valores citados nas tabelas americanas ou européias consultadas, expressam as variações existentes na composição corporal das raças estudadas, manejo alimentar, peso do animal e condições climáticas.

\section{CONCLUSÃO}

Pelos resultados obtidos, conclui-se que:

a) Houve diferença significativa $(\mathrm{p}<0,05)$ para os grupos F1 e para os animais puros Santa Inês sobre o conteúdo corporal do macromineral cálcio.

b) À medida que o peso corporal aumentou, houve redução no conteúdo corporal dos macrominerais cálcio e fósforo nos quatro grupos genéticos estudados.

c) Tabelas de exigências desenvolvidas com grupos genéticos e condições climáticas diferentes não refletem a real composição corporal e as exigências nutricionais em cálcio e fósforo de cordeiros puros Santa Inês e seus cruzamentos com Bergamácia, Ilê de France e Texel.

d) A estimativa da composição corporal por quilo de peso corporal vazio para animais de 15 a $45 \mathrm{~kg}$ de peso vivo foi: 14,641 a 11,637 $\mathrm{g}$ de Ca para os animais Santa Inês e 12,537 a 10,778 g de Ca para os demais grupos genéticos. No caso do fósforo, não houve dife- renças entre os grupos genéticos, e os valores variaram de 7,892 a 6,767 g de P para animais com 15 a $45 \mathrm{~kg}$ de PV.

e) As exigências líquidas de cordeiros SI variaram de 9,50 g a 7,56 $\mathrm{g}$ de Ca por $\mathrm{kg}$ de ganho de $\mathrm{PV}$ e de 5,50 g a 4,72 g de P por kg de ganho de PV; as exigências líquidas de cordeiros $\mathrm{BE}$, IF e TE variaram de $8,78 \mathrm{~g}$ a 7,54 $\mathrm{g}$ de Ca por $\mathrm{kg}$ de PV e de $5,50 \mathrm{~g}$ a $4,72 \mathrm{~g}$ de $\mathrm{P}$ por kg de ganho de PV.

\section{REFERÊNCIAS BIBLIOGRÁFICAS}

AGRICULTURAL AND FOOD RESEARCH COUNCIL. A repraisal of the calcium and phosphorus requierementsof sheep and cattle: report 6 . Nutrition Abstract review, series B, Cambridge, v. 61, n. 9, p. 573-612, 1991.

AGRICULTURAL RESEARCH COUNCIL. The nutrient reguirements of farm livestock. London, 1980. $351 \mathrm{p}$.

EUClideS, R. F.; SILVA, M. A. Manual de utilização do programa AVRPOL: análise de variância e regressão polinomial. Viçosa: UFV, 1979. $11 \mathrm{p}$.

GERASEEV, L. C.; PEREZ, J. R. O.; PRADO, O. V.; RESENDE, K. T.; SILVA FILHO, J. C.; BONAGURIO, S. Composição corporal e exigências nutricionais em cálcio e fósforo para o ganho e mantença de cordeiros Santa Inês dos $15 \mathrm{~kg}$ aos $25 \mathrm{~kg}$ de peso vivo. Revista da Sociedade Brasileira de Zootecnia, São Paulo, v. 29, n. 1, p. 261-268, 2000.

McDOWELL, L. R. Minerais para ruminantes sob pastejo em regiões tropicais: enfatizando o Brasil. 3 . ed. Gainesville: Universidade da Flórida, 1999. 93 p.

NATIONAL RESEARCH COUNCIL. Nutrient regueriments of domestic animals: nutríent requeriments of sheep. Washington, $1985.99 \mathrm{p}$.

PAUlinO, M. F.; FONTES, C. A. de A.; JORGE, A. M.; QUEIROZ, A. C. de; SILVA, J. F. C. da; GOMES JUNIOR, P. Composição corporal e exigências de macroelementos minerais ( $\mathrm{Ca}, \mathrm{P}, \mathrm{Mg}, \mathrm{Na}$ e $\mathrm{K}$ ) de bovinos não-castrados de quatro raças zebuínas. Revista Brasileira de Zootecnia, São Paulo, v. 28, n. 3, p. 634-641, 1999.

RESENDE, K. T. Métodos de estimativa da composição corporal e exigências nutricionais de proteína,

Ciênc. agrotec., Lavras. V.27, n.6, p.1370-1379, nov./dez., 2003 
energia e macrominerais inorgânicos de caprinos em crescimento. 1989. 130 f. Tese (Doutorado em Zootecnia) - Universidade Federal de Viçosa, Viçosa, 1989.

RIBEIRO, S. D. A. Composição corporal e exigências em proteína, energia e macrominerais de caprinos mestiços em fase inicial de crescimento. 1995. $100 \mathrm{f}$. Dissertação (Mestrado em Zootecnia) - Universidade Estadual Paulista, Jaboticabal, 1995.

SILVA, D. J. Análise de alimentos: métodos químicos e biológicos. Viçosa: UFV, 1998. 165 p.

SILVA, J. F. C. da. Exigências de macroelementos inorgânicos para bovinos: o sistema ARC/AFRC e a experiência no Brasil. In: SIMPÓSIO INTERNACIONAL SOBRE EXIGÊNCIAS NUTRICIONAIS DE RUMINANTES, 1995, Viçosa. Anais... Viçosa: UFV, 1995. p. 467-504.
SIGNORETTI, R. D. Consumo, digestibilidade, composição corporal, exigências nutricionais e eficiência de utilização da energia metabolizável para ganho de peso de bezerros holandeses. 1998. 157 f. Tese (Doutorado em Zootecnia) - Universidade Federal de Viçosa, Viçosa, 1998.

SNEDECOR, G. W.; COCHRAN, W. G. Statistical methods. 6. ed. Iowa: The Iowa State University, 1967. $593 \mathrm{p}$.

TRINDADE, I. A. C. M. Composição corporal e exigências nutricionais em macrominerais de ovinos lanados e deslanados, em crescimento. 2000. 66 f. Dissertação (Mestrado em Zootecnia) - Universidade Estadual Paulista, Jaboticabal, 2000. 